\subsection{3 \\ Étude des Effets de la Viscosupplémentation sur la Marche des Patients Arthrosiques}

JB. Candelier* (1) ; F. Ponchel (2) ; B. Auvinet (3) ; G. Jelin (1) ; P. Fardellone (1); V. Goeb (1)

(1) Rhumatologie, CHU Amiens-Picardie, Amiens ; (2) Rheumatology, Chapel Allerton Hospital, Leeds, Royaume Uni ; (3) Service de Rhumatologie, Polyclinique du Maine, Laval

*Auteur correspondant : jeanbaptiste.candelier@gmail.com (JB. Candelier)

Introduction. - L'objectif de cette étude est de déterminer si un test de marche par accéléromètrie est un marqueur objectif de la douleur chez les patients arthrosiques traités par injection intra articulaire d'acide hyaluronique.

Matériel et Méthodes. - Nous avons réalisé une étude prospective, monocentrique, observationnelle, exploratoire chez 37 patients présentant une gonarthrose ou une coxarthrose douloureuse. Un test de marche par accélérométrie sur 30 mètres a été réalisé avant et 1 mois après une injection intra articulaire d'acide hyaluronique. Les données accéléromètriques relevées étaient : vitesse, régularité, symétrie, onde de choc pathogène et puissance totale. L'échelle visuelle analogique douleur (EVA) et l'indice de Lequesne ont également été mesurés à l'inclusion et à 1 mois.

Résultats. - Les données démographiques (âge, sexe, IMC), l'EVA et l'indice de Lequesne n'avaient pas d'impact sur les données accélérométriques. Le choix d'une spécialité pharmacologique n'influençait pas les résultats du test accélérométrique. La diminution de l'EVA est associée à une augmentation de la vitesse $(\mathrm{p}=0,048)$. On observe une tendance d'association avec l'onde de choc pathogène $(\mathrm{p}=0,056)$. Une réponse en terme de régularité est associé à une amélioration de l'indice de Lequesne ( $p=0,013)$. Une amélioration de la symétrie est associée à une réduction de l'EVA $(p=0,048)$ et à une amélioration de l'indice de Lequesne ( $p=0,002)$.

Discussion. - Une réponse en terme de régularité est associée à une amélioration de l'indice de Lequesne ( $p=0,013)$, une amélioration de la symétrie est associée à une réduction de la douleur $(\mathrm{p}=0,048)$ et à une amélioration de l'indice de Lequesne $(\mathrm{p}=0,002)$. Un test de marche par accélérométrie pourrait ainsi représenter un marqueur objectif de l'intensité de la douleur. Auvinet et al. [1] ont démontré la présence des troubles de la marche importants chez des patients atteints de fibromyalgie retrouvant comme dans notre étude une association entre trouble de la marche et pathologie douloureuse. Tang et al. [2], en utilisant une méthode basée sur l'analyse des amplitudes articulaires par 6 caméras, retrouve une amélioration de la flexion et de l'extension du genou suite à l'usage d'injection intra articulaire d'acide hyaluronique chez les patients souffrant de gonarthrose. Ces résultats pourraient se traduire en accélérométrie par l'amélioration de la régularité et de la symétrie observée dans notre étude. La force de cette étude réside dans son caractère prospectif et exploratoire, avec un recrutement pragmatique. Il s'agit en effet de la première étude explorant le test de marche par accélérométrie comme potentiel marqueur de la douleur chez les patients arthrosiques. Du fait de la courte durée de suivi de cette pathologie chronique et du faible nombre de patients inclus, cette étude nécessite des résultats à une plus grande échelle avant de pouvoir conclure sur des bases solides

Conclusion. - La régularité et la symétrie mesurées par accélérométrie sont des marqueurs objectifs de la douleur chez les patients arthrosiques traités par injection intra articulaire d'acide hyaluronique. D'autres études de plus grande ampleur et avec un suivi plus long seront nécessaires afin de confirmer ces résultats.

Conflit d'intérêt. - aucun

Références bibliographiques

[1] Auvinet B, Bileckot R, Alix A-S, et al. Les troubles de la marche chez le patient atteint de fibromyalgie. Revue Du Rhumatisme 2006;73:932-5.
[2] Tang AC-W, Tang SF-T, Hong $W-H$, et al. Kinetics features changes before and after intra-articular hyaluronic acid injections in patients with knee osteoarthritis. Clin Neurol Neurosurg 2015; 129 Suppl 1 : S21-26.

\subsection{4}

\section{Recommandations du groupe EUROVISCO pour le retraitement par acide hyaluronique des patients préalablement traités par viscosupplémentation pour une gonarthrose}

X. Chevalier (1) ; H. Bard (2) ; A. Migliore (3) ; Y. Henrotin (4); J. Monfort (5) ; T. Conrozier* (6) ; R. Raman (7) ; D. Baron (8) ; J. Jerosch (9); P. Richette (10)

(1) Rhumatologie, Hôpital Henri Mondor, Créteil ; (2) Rhumatologie, Hôpital Européen Georges-Pompidou, Paris ; (3) U.o.s. of rheumatology, Ospedale San Pietro Fatebenefratelli, Rome, Italie ; (4) Arthropôle Liège, Université de Liège, Liège, Belgique; (5) Servei de reumatologia, Hospital del Mar, Barcelone, Espagne ; (6) Rhumatologie, Hôpital Nord Franche-Comté, Belfort ; (7) Academic department of orthopaedics, Hull and East Yorkshire NHS Trust Castle Hill Hospital, Cottingham, Royaume Uni ; (8) Rhumatologie, Hopital de Lannion-Trestel, TrévouTréguignec ; (9) Orthopedic department, Johanna-Etienne-Hospital, Neuss, Allemagne ; (10) Inserm umr1132, Hôpital Lariboisière, Paris *Auteur correspondant : thierry.conrozier@hnfc.fr (T. Conrozier)

Introduction. - L'objectif de ce travail était de proposer aux médecins pratiquant la viscosupplémentation (VS), un arbre décisionnel sur la prise de décision de retraitement chez les patients préalablement traités par des injections d'acide hyaluronique $(\mathrm{AH})$ pour une gonarthrose

Matériel et Méthodes. - Dix experts de l'arthrose, rhumatologues et chirurgiens orthopédiques, de 6 pays Européens, se sont réunis dans le but de rédiger un algorithme de décision pour le retraitement par VS chez les patients ayant déjà été traités par injections d'AH. Une revue de la littérature exhaustive a été réalisée et 88 questions ont été posées aux participants. Pour chacune d'entre elle l'expert devait se prononcer à l'aide d'une échelle en 4 points (1-Pas du tout d'accord, 2-plutôt pas d'accord, 3-plutôt d'accord, 4-tout à fait d'accord). Après discussion une position commune était retenue par le groupe.

Deux situations ont été distinguées: Retraitement après échec d'une $1^{\text {re }}$ VS et retraitement après succès d'une précédente VS. Le premier point a donc été de définir les critères d'échec et de succès. Pour conclure le groupe de travail a fait des propositions sur la conduite à tenir en cas d'effets indésirables (EI) induits par le précédent traitement et a analysé l'intérêt et les limites de l'utilisation des biomaqueurs comme aide à la prise de décision.

Résultats. - Parmi tous les critères permettant de retenir la notion d'échec ou de succès, le PASS (Patient's Acceptable Symptom State) et l'avis du patient ont fait l'objet d'un consensus, contrairement à la variation du WOMAC ou d'autres scores conseillés seulement pour le suivi des essais cliniques. En cas d'échec avéré le groupe de travail a insisté sur une démarche diagnostique rigoureuse (l'indication était elle bonne, existe-t-il des facteurs prédictifs d'échec, le protocole thérapeutique a-t-il été respecté, peut on assurer que les injections étaient intra-articulaires ?). Pour chacune de ces questions des propositions ont été avancées. Chez les patients préalablement traités avec succès, la question principale est celle du moment optimal pour envisager des nouvelles injections. Schématiquement, un retraitement ne doit pas être envisagé chez les patients asymptomatiques sauf cas particulier (fort risque de progression, sujets très jeunes, sportifs professionnels, prothèse contre-indiquée). Chez les patients peu symptomatiques dont les douleurs ne s'aggravent pas, un retraitement doit être proposé à 1 an sauf avis contraire du patient ou absence de facteur de risque d'évolution. En cas d'augmentation des douleurs la décision de retraitement repose sur l'avis 
du patient et le PASS. Un retraitement le plus précoce possible est conseillé. En cas d'EI non sévère lors de la précédente viscosupplémentation il n'est pas nécessaire de changer de viscosupplément mais l'usage d'un corticoïde ou d'un anti-inflammatoire a été proposé, sans qu'un consensus n'ait pu être établi. Enfin, si l'usage de biomarqueurs est recommandé au cours des essais cliniques, le niveau de preuve est encore insuffisant pour les utiliser comme aide à la décision.

Conclusion. - Les deux arbres de décision sont destinés à aider les praticiens dans leur prise de décision chez les patients souffrant de gonarthrose et déjà traités par viscosupplémentation.

Conflit d'intérêt. - aucun

\section{POLYARTHRITE RHUMATOÏDE}

\subsection{5}

\section{Comparaison du certolizumab pegol versus adalimumab : résultats d'efficacité et de tolérance à 2 ans d'une étude comparative directe de supériorité, en aveugle pour les investigateurs}

R. Chapurlat* (1) ; R. Fleischmann (2) ; G. Burmester (3) ; B. Combe (4) ; J. Curtis (5) ; S. Hall (6) ; B. Haraoui (7) ; R. Van Vollenhoven (8) ; C. Cioffi (9) ; C. Ecoffet (10) ; L. Ionescu (10) ; L. Gervitz (11) ; L. Peterson (12) ; JS. Smolen (13)

(1) Pavillon $f$, hôpital e. herriot, INSERM UMR1033 et Université de Lyon, Lyon ; (2) Centre de recherche clinique metroplex, Centre Médical SW de l'Université du Texas, Dallas, États-Unis ; (3) Rhumatologie et immunologie, Université de Médecine Charité, Berlin, Allemagne ;

(4) Service d'Immuno-Rhumatologie, C.H.U. Lapeyronie, Montpellier ;

(5) Rhumatologie, The University of Alabama, Birmingham ;

(6) Rhumatologie, Université Monash, Melbourne, Australie ;

(7) Rhumatologie, Institut De Rhumatologie De Montreal, Montreal, Canada ; (8) Rhumatologie, Institut Karolinska, Stockholm, Suède ; (9) Développement clinique immunologie, UCB Biosciences Inc, Raleigh, États-Unis ; (10) Médical immunologie, UCB Pharma, Anderlecht, Belgique ; (11) Medical, UCB Pharma, Raleigh, États-Unis ;

(12) Medical, UCB Biosciences Inc, Raleigh, États-Unis ; (13) Division of rheumatology, Medizinische Universität Wien, Vienne, Autriche *Auteur correspondant : roland.chapurlat@inserm.fr (R. Chapurlat)

Introduction. - Les comparaisons directes des traitements de fond biologiques (bDMARDs) dans le traitement de la PR devraient fournir des preuves rigoureuses de l'efficacité comparative de différents traitements. S'il existe plusieurs études comparatives directes entre anti-TNF et bDMARDs ayant des mécanismes d'action différents [1-3], aucune étude comparative directe de l'efficacité et de la tolérance des bDMARDS au sein de la même classe, y compris les anti-TNF, n'a été rapportée.

Patients et Méthodes. - L'étude EXXELERATE était une étude comparative directe de supériorité de 104 semaines, en groupes parallèles, en aveugle pour les investigateurs, comparant l'efficacité et la tolérance à court terme $(\mathrm{S} 12)$ et à long terme $(\mathrm{S} 104)$ du certolizumab pegol (CZP)+MTX versus adalimumab (ADA)+MTX (Figure). Les patients (pts) ont été randomisés selon un ratio 1:1 pour recevoir CZP+MTX ou ADA+MTX. À S12, les pts ont été classés comme répondeurs (DAS28 [VS] $\leq 3,2$ ou réduction du DAS28 [VS] $\geq 1,2$ par rapport à l'inclusion à S12) ou non-répondeurs (NR). Les NR à l'un des anti-TNF ont changé pour l'autre anti-TNF (Figure). Les critères primaires étaient le pourcentage de pts répondeurs ACR20 à S12 et en faible activité de la maladie (low disease activity, LDA; DAS28 [VS] $\leq 3,2$ ) à S104 (les NR à S12 ont été considérés comme NR pour la LDA). Les critères secondaires incluaient la proportion de pts répondeurs ACR20 à S6, en LDA (DAS28 [VS]) à S6, S12, S52 et la proportion des pts répondeurs à S12 en LDA à S104. Les critères exploratoires incluaient la proportion des pts répondeurs ACR20/ 50/70 à S12 et en LDA et rémission (REM) (DAS28 [VS] et CDAI) à chaque visite.

Résultats. - À l'inclusion, 915 pts ont été randomisés pour recevoir soit CZP+MTX $(n=457)$, soit ADA+MTX ( $n=458)$. À S12, 359 pts CZP+MTX (78,6\%) et 369 pts ADA+MTX (80,6\%) étaient répondeurs. Aucune différence statistiquement significative n'a été observée pour la réponse ACR20 à S12 (69,2\% et 71,4\%; odds ratio (OR) : 0,90 [IC à $95 \%: 0,67 ; 1,20]$ ]) et la LDA (DAS28 [VS]) à S104 $(35,5 \%$ et $33,5 \%$; OR : 1,09 [IC à $95 \%: 0,82 ; 1,45$ ]) entre CZP+MTX et ADA+MTX, respectivement. Aucune différence entre les bras de traitement n'a été observée pour les critères secondaires et exploratoires d'efficacité (Tableau). Une proportion similaire d'événements indésirables apparus sous traitement (TEAEs; $75,4 \%$ et $73,8 \%$ ), de TEAEs graves $(13,0 \%$ et $11,1 \%$ ) et d'infections et infestations graves $(3,3 \%$ et $3,1 \%$ ) a été rapportée dans les bras CZP+MTX et ADA+MTX, par traitement à la survenue de l'EI (taux d'événements pour 100 pt-année 257,5 versus 260,0 ).

Tableau Réponses cliniques (\% de patients)

\begin{tabular}{|c|c|c|c|c|}
\hline & & $\mathrm{CZP}+\mathrm{MTX}$ & $\mathrm{ADA}+\mathrm{MTX}$ & $\begin{array}{c}\text { Odds ratio } \\
\text { comparant } \\
\text { le CZP à ADA } \\
\text { (référence) } \\
\text { (IC95\%) }\end{array}$ \\
\hline $\begin{array}{l}\text { Variables d'effica- } \\
\text { cité à court terme } \\
\text { (FAS) }\end{array}$ & & $\mathrm{n}=454$ & $n=454$ & - \\
\hline ACR20 & S6 & 64,5 & 60,8 & $\begin{array}{c}1,21(0,92 \\
1,59)\end{array}$ \\
\hline \multirow[t]{2}{*}{ ACR20 } & S12 [a] & 69,2 & 71,4 & $\begin{array}{c}0,90(0,67 \\
1,20)\end{array}$ \\
\hline & S6 & 20,5 & 18,1 & $\begin{array}{c}1,15(0,81 \\
1,62)\end{array}$ \\
\hline LDA (DAS28 [VS]) & S12 & 30,4 & 29,7 & $\begin{array}{c}1,00(0,75 \\
1,34)\end{array}$ \\
\hline \multicolumn{5}{|l|}{$\begin{array}{l}\text { Variables } \\
\text { d'efficacité à long } \\
\text { terme (FAS) }\end{array}$} \\
\hline $\begin{array}{l}\text { Les patients } \\
\text { n'ayant pas } \\
\text { atteint la LDA, } \\
\text { sortaient de } \\
\text { l'étude ou } \\
\text { changeaient } \\
\text { de traitement }\end{array}$ & & $\mathrm{n}=454$ & $\mathrm{n}=454$ & - \\
\hline \multirow[t]{2}{*}{ LDA (DAS28 [VS]) } & S52 & 41,6 & 38,3 & $\begin{array}{c}1,15(0,87 \\
1,51)\end{array}$ \\
\hline & S104 [a] & 35,5 & 33,5 & $\begin{array}{c}1,09(0,82 \\
1,45)\end{array}$ \\
\hline \multirow[t]{3}{*}{$\begin{array}{l}\text { Répondeurs à S12 } \\
\text { (FASS S12) }\end{array}$} & & $n=353$ & $n=361$ & - \\
\hline & $\mathrm{S} 12$ & 86,1 & 85,9 & \\
\hline & $\mathrm{S} 24$ & 85,6 & 85,0 & \\
\hline \multirow[t]{3}{*}{ ACR20 } & S52 & 77,9 & 79,5 & - \\
\hline & S104 & 64,9 & 66,8 & \\
\hline & $\mathrm{S} 12$ & 51,3 & 53,2 & \\
\hline \multirow[t]{4}{*}{ ACR50 } & S24 & 63,5 & 62,6 & - \\
\hline & S52 & 60,6 & 64,0 & \\
\hline & S104 & 53,3 & 56,8 & \\
\hline & $\mathrm{S} 12$ & 28,0 & 26,9 & \\
\hline \multirow[t]{4}{*}{ ACR70 } & S24 & 39,1 & 40,4 & - \\
\hline & S52 & 43,6 & 43,5 & \\
\hline & S104 & 39,7 & 41,3 & \\
\hline & $\mathrm{S} 12$ & 38,5 & 36,8 & \\
\hline
\end{tabular}

